\title{
Attachment and mentalization as predictors of outcome in family therapy for adolescent anorexia nervosa
}

\author{
Tom Jewell ${ }^{1,2}\left(\right.$ Moritz Herle $^{3} \cdot$ Lucy Serpell $^{4,5} \cdot$ Alison Eivors $^{6} \cdot$ Mima Simic $^{7} \cdot$ Peter Fonagy $^{5,8} \cdot$ Ivan Eisler $^{7}$
}

Received: 11 August 2021 / Accepted: 17 December 2021

(c) The Author(s) 2021

\begin{abstract}
Anorexia nervosa-focussed family therapy (FT-AN) is the first-line treatment for adolescent anorexia nervosa (AN), but the predictors of poor treatment response are not well understood. The main aim of this study was to investigate the role of attachment and mentalization in predicting treatment outcome. The secondary aims of the study were to investigate therapeutic alliance at 1 month as a predictor of outcome, and to test the associations between alliance and baseline attachment and mentalization. 192 adolescents with AN and their parents were recruited as they began family therapy in out-patient specialist eating disorder services. Self-report measures of attachment, mentalization, and emotion regulation were completed at the start of treatment by adolescent patients and one of their parents. Self-reported alliance scores were collected at one month. Higher scores on the Certainty Scale of the Reflective Functioning Questionnaire, completed by parents, which indicate over-certainty about mental states, were the strongest predictor of poor outcome (Odds Ratio: 0.42, CI: 0.20-0.87). Similarly, for adolescents, higher Lack of Clarity scores on the Difficulties in Emotion Regulation Scale, representing being unclear about one's feelings, were predictive of positive treatment outcome (OR: 1.10, CI: 1.00-1.21). Higher alliance scores at 1 month predicted positive outcome, and were associated with attachment security and mentalization. These novel findings suggest that, particularly in parents, a tendency towards excessive certainty about mental states in others may predict poor outcome in FT-AN. Further research is warranted to replicate the finding and characterise families at risk of poor outcome.
\end{abstract}

Keywords Attachment $\cdot$ Mentalization $\cdot$ Family therapy $\cdot$ Anorexia nervosa $\cdot$ Therapeutic alliance

\section{Introduction}

Tom Jewell

tom.1.jewell@kcl.ac.uk

1 Florence Nightingale Faculty of Nursing, Midwifery and Palliative Care, King's College London, London, UK

2 Great Ormond Street Hospital NHS Foundation Trust, London, UK

3 Department of Biostatistics and Health Informatics, King's College London, London, UK

4 North East London NHS Foundation Trust, London, UK

5 Research Department of Clinical, Educational and Health Psychology, University College London, London, UK

6 Leicestershire Partnership NHS Foundation Trust, Leicester, UK

7 South London and Maudsley NHS Foundation Trust, London, UK

8 Anna Freud Centre, London, UK
Anorexia nervosa (AN) is a serious condition which typically emerges during adolescence. AN is associated with high rates of psychiatric co-morbidity [1] and significantly raised mortality rates [2], with an estimated incidence of 14 per 100,000 [3]. Specialist anorexia nervosa-focussed family therapy for children and young people (FT-AN) has the strongest efficacy evidence in the treatment of adolescent AN and was recommended by NICE [4] as the first-line treatment. However, a significant proportion of patients fail to respond and a lack of evidence on predictors of treatment response and understanding of mechanisms of change limits efforts to personalise treatment recommendations. There is therefore an urgent need for well-designed and sufficiently powered research that can investigate predictors of response and change processes in FT-AN [5].

Currently, the most robust findings relating to treatment response in FT-AN relate to clinical indicators, with shorter duration of illness and higher percentage body mass index 
at start of treatment being associated with more positive outcomes (see Jewell et al. [6] for a review). In terms of early markers, early weight gain [7] and positive ratings of the therapeutic alliance are predictive of good treatment response in FT-AN [8]. In terms of relational variables, there is evidence that parental expressed emotion is associated with poorer outcome [9], but there is almost no research on how adolescent psychological characteristics beyond features of eating pathology might impact treatment outcome.

The present study sought to shed light on predictors of outcome and mechanisms of change in FT-AN by focussing on two constructs that are salient in eating disorders: attachment and mentalizing. Attachment can be understood as a broad, higher order construct which has been operationalized in multiple ways, with a foundation in the conceptual and empirical work of Bowlby [10] and Ainsworth [11]; attachment style, the operationalization used in this study, refers to a constellation of knowledge, expectations, and insecurities that people hold about themselves and their close relationships [12]. Meta-analytic evidence suggests that individuals with eating disorders have higher rates of insecure attachment, and more difficulties mentalizing, than community controls $[6,13]$. Moreover, attachment is an established predictor of both outcome and therapeutic alliance in adult psychotherapy [14, 15]. In adult eating disorders, attachment security has been shown to predict positive therapeutic alliance [16] and differential response to treatment [17], including rates of dropout.

Mentalization refers to the capacity to understand others' actions as well as one's own behaviour in terms of intentional mental states, such as feelings, desires, attitudes, and goals, and is assumed to predict the capacity for emotion regulation. Reflective function refers to this ability operationalized in the context of attachment relationships [18]. Difficulties in reflective function can be conceptualised as falling into two broad domains: hypomentalizing refers to an inability to consider complex models of one's own mind and/ or that of others, whereas hypermentalizing involves making unjustified assumptions about other people's mental states that go far beyond the observable data [19]. Mentalization has been shown to predict alliance [20] and outcomes [21] in adults with eating disorders.

\section{Proposed links between attachment, mentalization, and treatment outcome}

A person's ability to mentalize effectively is a dynamic capacity that is influenced by stress and arousal, particularly in the context of attachment relationships [19]. Under conditions of high arousal, patterns of brain activity 'switch' from flexibility to automaticity [22], leading to pre-mentalizing modes of experiencing subjectivity, such as over-certain modes of thought [23]. Attachment security, which raises the threshold for this switch-point to automatic/pre-mentalizing modes of thought, can thus be conceptualised as a protective factor for individuals experiencing affectively charged occurrences within their attachment network. We therefore hypothesised that attachment security and capacity for mentalization would be associated with positive treatment outcome in FT-AN, given that the process of attending treatment for, and recovering from, $\mathrm{AN}$ is associated with high levels of stress for adolescents and their families [24].

Attachment security was postulated as a relational strength that could increase the likelihood of forming a positive therapeutic alliance. By contrast, insecure attachment may make family members vulnerable to experiencing prementalizing modes of thought in the context of emotionally charged situations. Within this model, emotional regulation might also emerge as a predictor of outcome, given its theoretical and empirical associations with attachment and mentalizing [25]. We proposed that insecure attachment, mentalizing difficulties, and emotional dysregulation at the start of treatment would be associated with poorer therapeutic alliance ratings at one month. We hypothesised that one mechanism by which attachment and mentalization might influence outcome is via their association with the development of therapeutic alliance, a well-established predictor of outcome in eating disorders and psychotherapy [26, 27]. Our theoretical model is depicted visually in Fig. 1.

Hypotheses The aim of the study is to address the following hypotheses:

(1) Baseline insecure attachment, mentalizing difficulties, and emotion regulation difficulties, in both adolescents and parents, will predict categorical poor treatment outcome at nine months.

(2) Positive ratings of therapeutic alliance at one month by adolescents and parents will predict good categorical treatment outcome at nine months.

(3) Secure attachment, better mentalizing, and lower emotion regulation difficulties at baseline will predict positive therapeutic alliance at one month for parents and adolescents.

\section{Secondary analysis}

We will also conduct a secondary analysis to test the following hypothesis: insecure attachment, mentalizing difficulties, and emotion regulation difficulties, assessed at baseline in both adolescents and parents will predict lower gains in percentage median body mass index (\%mBMI) at 9 months. 


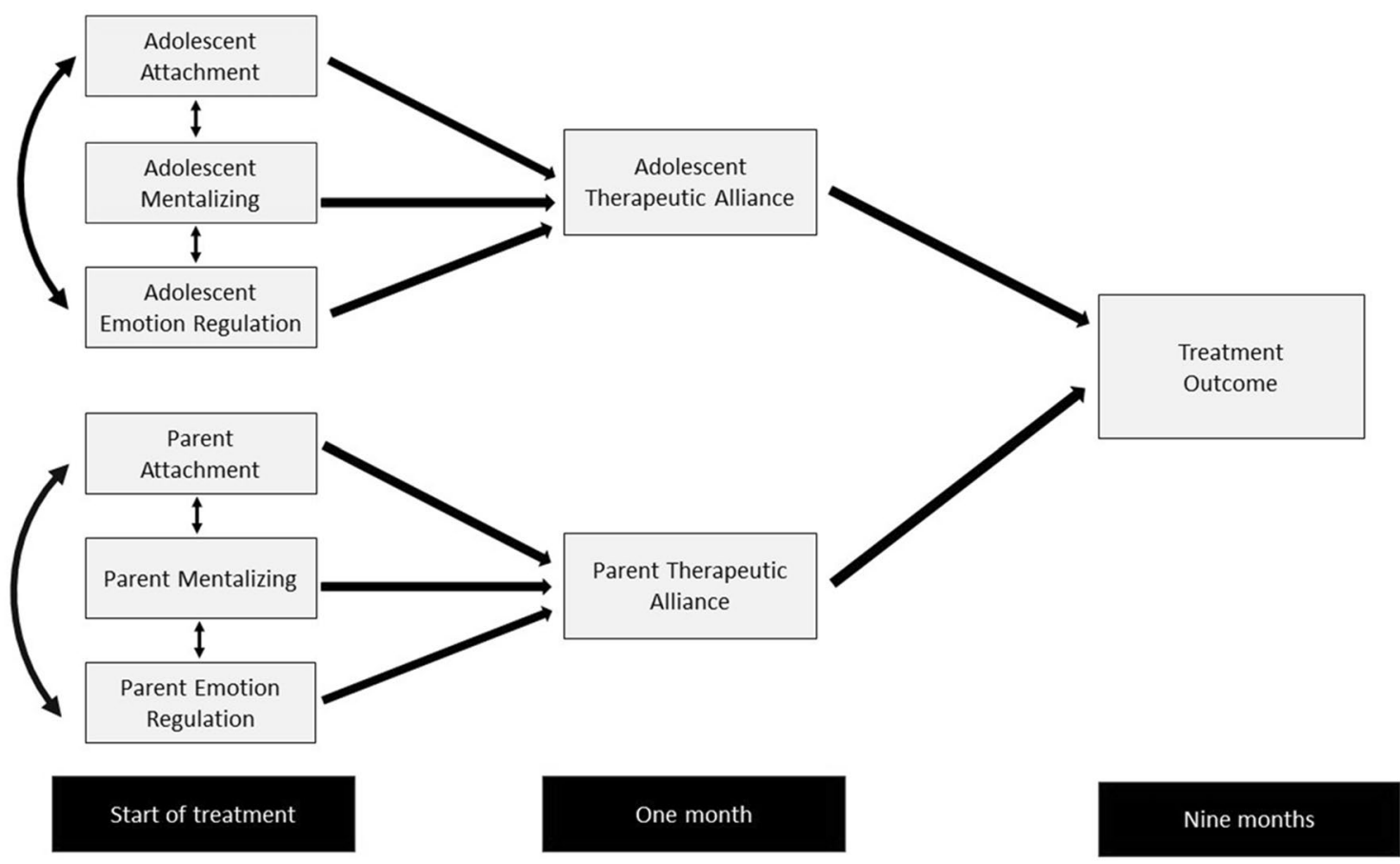

Fig. 1 Conceptual diagram showing hypothesised model. Double headed arrows refer to correlations between variables

\section{Methods}

Data were collected from three clinical sites in the United Kingdom, all of which are specialist community-based eating disorder services based in two diverse, multi-ethnic cities. Adolescent patients and their families meeting criteria for the study were approached at assessment or start of treatment. All consecutive cases meeting eligibility within the data collection period were invited to participate. Patient inclusion criteria were as follows: aged between 10 and 17; living with their parent/s or carer/s for at least the previous 3 months; diagnosed at clinical assessment with anorexia nervosa (restricting or binge/purge sub-type) using DSM-V criteria [28]; or meeting DSM-V criteria for Other Specified Feeding or Eating Disorder (OSFED) providing their $\% \mathrm{mBMI}$ at assessment was at $85 \%$ or below, or the adolescent had lost $15 \%$ of their body weight in the 3 months prior to assessment; adequate level of English, i.e., sufficient to understand study information sheets and consent forms; receiving out-patient family therapy for anorexia nervosa (FT-AN) as their treatment. Parent/ carer criteria were that they would be involved in attending FT-AN sessions and had an adequate level of English. At least one parent/carer needed to consent to take part in the study. Up to two parents/carers could be recruited per adolescent.

\section{Data collection}

Self-report measures of attachment, mentalization, and emotion regulation were completed at the start of treatment (T1) and 1 month into treatment (T2) by adolescent patients and at least one of their parents. At T2 self-report measures of alliance were also completed by parents and adolescents.

Young people and parents completed self-report measures online using the Bristol Online Survey, usually from their own homes (approximately $5 \%$ of $\mathrm{T} 1$ questionnaires were completed on paper). Weight and height data were collected by clinicians as part of the routine clinical assessment. The conversions to percent median body mass index (\% mBMI) were done using a computer programme based on the Child Growth Foundation [29] development charts.

\section{Outcomes}

Primary outcome: Morgan-Russell scales as modified by Russell et al. [30] in which categorical outcome was recorded at 9 months at one of three levels, as follows:

Good outcome-participants whose weight is above $85 \% \mathrm{mBMI}$, who are menstruating and have no bulimic symptoms; Intermediate outcome-participants meet the same weight criteria, but are either not menstruating or have occasional bulimic symptoms (averaging less than 
once a week over the past month); Poor outcome-participants whose weight is below $85 \% \mathrm{mBMI}$ or have developed bulimic symptoms more than once a week. Those in inpatient care at 9 months were also conservatively classified as having a poor outcome.

Weight data are reported as a percentage of median BMI (\% mBMI), adjusted for height, age, and sex.

\section{Adolescent predictor variables at Time 1}

- Attachment Style Questionnaire (ASQ) [31]: This 40-item attachment measure was developed for use with both adults and adolescents and is comprised of five subscales: Confidence, Preoccupation with Relationships, Need for Approval, Relationships as Secondary, and Discomfort with Closeness. Internal reliability and test-retest reliability are both good.

- Difficulties in Emotion Regulation Strategies Scale (DERS) [32]: This 36-item measure assesses emotion regulation difficulties and is comprised of six subscales: Nonacceptance of Emotional Responses, Difficulties Engaging in Goal-directed Behaviours, Impulse Control Difficulties, Lack of Emotional Awareness, Limited Access to Effective Emotion Regulation Strategies, and Lack of Emotional Clarity. The measure has demonstrated high internal consistency and good construct and predictive validity in clinical and non-clinical populations.

- Hypermentalizing Questionnaire-Adolescent version (parent scale) (Sharp, Barr and Vanwoerden (2018) Unpublished Manuscript): This scale comprises 26 items which assess adolescent hypermentalizing in their interaction with parents (e.g., "I worry a lot about what my parents are thinking and feeling"). The measure yields a total Hypermentalizing score, in which higher scores represent greater levels of hypermentalizing. Validity for the scale comes from an unpublished study in which the measure demonstrated convergent validity with the hypermentalizing scale of the Movie for Assessing Social Cognition [33], an experimental measure of mentalizing. The measure has been validated in an adolescent psychiatric sample comprised predominantly of youths with a diagnosis of Borderline Personality Disorder.

- Reflective Function Questionnaire-Youth (RFQY) [34]: This 46-item measure yields a Total score, with higher scores representing greater reflective function (sample item: "I always know what I feel"). The RFQY has adequate internal reliability. Convergent, criterion, discriminant, and construct validity have been demonstrated in an adolescent inpatient psychiatric sample, of which a small minority of participants had an eating disorder.

\section{Parent predictor variables at Time 1}

- Reflective Function Questionnaire (RFQ8) [35]: This 8-item measure yields two subscales: Certainty about Mental States (henceforth referred to as Certainty) and Uncertainty about Mental States (henceforth: Uncertainty). Scoring is applied, such that extreme scores at either end of the Likert scale are indicative of inadequate mentalizing. For instance, strong agreement with the item "I always know what I feel" yields a higher score on the Certainty scale, whereas strong agreement with the item "I don't always know why I do what I do" yield a higher score on the Uncertainty scale. The measure has demonstrated satisfactory levels of internal validity, convergent validity, and test-retest reliability.

- ASQ

- DERS

- Hypermentalizing Questionnaire-Parent version (adolescent scale): Parents completed the adolescent scale of the HMZ, which assesses parental hypermentalizing in their interaction with their adolescent (e.g., "My adolescent often says I over interpret his/her behavior or words").

\section{Adolescent and parent Time 2 predictor variables}

- System for Observing Family Therapy Alliance-Selfreport (SOFTA) [36]: This 16-item measure assesses perceptions of family therapy alliances on four subscales: Engagement in the Therapeutic Process, Emotional Connection to the Therapist; Safety within the Therapeutic System and Shared Sense of Purpose within the Family. Internal reliability for the total scale is good, and adequate-to-good for the four subscales. Convergent validity with an observer-rated version of the SOFTA has been demonstrated.

\section{Baseline covariates}

- Eating Disorder Examination Questionnaire (EDE-Q) [37]: This 28-item measure of eating disorder pathology has good internal consistency, test-retest reliability, and discriminant validity. The EDE-Q Global Score completed at initial clinical assessment was extracted from patient records.

- Baseline percentage median Body Mass Index (\%mBMI) adjusted for age and gender.

- Duration of illness in months. This refers to the number of months that the young person had been unwell for prior to their assessment in clinic.

EDE-Q was completed by adolescents at assessment (prior to entry to the study). Data on baseline percentage 
BMI and duration of illness were extracted from clinical notes from the time of clinical assessment.

\section{Baseline measures of co-morbidity}

- Revised Child Anxiety and Depression Scale (RCADS) Youth self-report version [38]: This 47-item questionnaire has good-to-excellent internal consistency, good test-retest reliability, and strong convergent and divergent validity. The Total Internalising Scale completed at initial clinical assessment was extracted from patient records.

- Self-harm at assessment-this was based on adolescent report at clinical assessment and was extracted from patient records, coded as 1 (for present) or 0 (not present).

\section{Study registration}

The study was pre-registered with Europe PubMed Central, with a brief overview of the study available at http:// europepmc.org/grantfinder/grantdetails?query $=$ pi $\% 3 \mathrm{~A} \%$ 22Jewell\%20T\%22\%20gid\%3A\%22CDRF-2014-05-024\% $22 \% 20$ ga $\% 3 \mathrm{~A} \% 22 \mathrm{National} \% 20$ Institute $\% 20$ for $\% 20 \mathrm{Health} \%$ 20Research $\% 20 \% 28$ NIHR\%29\%22\&cat=.

\section{Ethics}

The study was approved by the Camden and King's Cross ethics committee of the Health Research Authority.

\section{Sample size}

The study aimed to recruit 200 participants. The power calculation assumptions were that a sample size of 200 with follow-up of $80 \%$ at 9 months and 30\% experiencing poor treatment outcome would have $80 \%$ power to detect an odds ratio (OR) of $>1.58$ in relation to a one standard deviation difference in the predictor variable in the logistic regression analysis with an alpha level of 0.05 .

\section{Internal reliability}

We report McDonald's Omega for all self-report measures in Table 2. McDonald's Omega was chosen since it is considered a less biased metric of internal consistency than Cronbach's alpha. For the EDE-Q and RCADS data, we did not have item-level data available for our participants, as these scores were extracted from patient notes. Therefore, McDonald's Omega for these two measures were calculated from routine outcome databases from Site 1 .

\section{Public and patient involvement (PPI)}

Parents and adolescents with lived experience of AN were involved in the design and interpretation of this study. The choice of measures and methods for approaching and recruiting participants were informed by PPI feedback. To guide interpretation, a public meeting was held in August 2019 to share findings with those with lived experience, which was followed up by dissemination over email of lay summaries in late 2019. The findings were discussed over email and telephone with several individuals with lived experience. Suggestions from these activities are included in the discussion section.

\section{Analysis plan}

For the purposes of the main analysis, the primary outcome was a binary variable combining Good and Intermediate outcome to compare with Poor outcome on the Morgan Russell scales.

The analyses were conducted in two stages:

1. Binary logistic regression was used to test the association between predictors and therapy outcome at 9 months after treatment began.

2. Linear regression was used to test the associations between $\mathrm{T} 1$ and $\mathrm{T} 2$ predictors.

Robust standard errors were used in all analyses due to nonindependence of participants due to clustering by site. Alpha level was set at 0.05 . Predictor variables were tested at the subscale level, with total scores used only when subscale scores were unavailable.

All analyses included the following covariates recorded at the time of clinical assessment: age (in years); duration of illness (in months) at the time of assessment; severity of eating pathology using the EDE-Q Global score; baseline percentage median Body Mass Index adjusted for age and gender; and treatment site.

\section{Missing data}

Missing data for individual items on the self-report measures of predictor variables were low, since most questionnaires were completed online, with participants unable to submit their responses if items were missing. Approximately $5 \%$ of participants completed paper questionnaires at Time 1. Missing data were more frequent for Time 2 data. There were also higher rates of missing data for some baseline covariate measures, such as the RCADS and EDE-Q measures, which were drawn from data collected 
Table 1 Demographic characteristics of the sample

\begin{tabular}{|c|c|c|c|c|c|}
\hline & $N$ & Minimum & Maximum & Mean & SD \\
\hline Age & 173 & 11 & 17 & 14.69 & 1.54 \\
\hline$\%$ Median body mass index & 173 & 62.27 & 113.79 & 83.89 & 8.97 \\
\hline \multirow[t]{2}{*}{ Duration of illness (months) } & 170 & 1 & 65 & 10.48 & 10.59 \\
\hline & & & \multicolumn{2}{|l|}{$N$} & $\%$ \\
\hline \multicolumn{6}{|l|}{ Sex } \\
\hline \multicolumn{2}{|l|}{ Female } & & \multicolumn{2}{|l|}{153} & 88.4 \\
\hline Male & & & \multicolumn{2}{|l|}{20} & 11.6 \\
\hline \multicolumn{6}{|l|}{ Lives with } \\
\hline Natural/adoptive parents & & & \multicolumn{2}{|l|}{129} & 74.6 \\
\hline Mother alone & & & \multicolumn{2}{|l|}{24} & 13.9 \\
\hline Other & & & \multicolumn{2}{|l|}{20} & 11.6 \\
\hline \multicolumn{6}{|l|}{ Ethnicity } \\
\hline White British & & & \multicolumn{2}{|l|}{138} & 79.8 \\
\hline Other White & & & \multicolumn{2}{|l|}{13} & 7.5 \\
\hline Dual Heritage & & & \multicolumn{2}{|l|}{10} & 5.8 \\
\hline British Asian/Other South & & & \multicolumn{2}{|l|}{9} & 5.2 \\
\hline Other & & & \multicolumn{2}{|l|}{3} & 1.7 \\
\hline \multicolumn{6}{|l|}{ Diagnosis } \\
\hline Anorexia nervosa (restricti & & & \multicolumn{2}{|l|}{140} & 80.9 \\
\hline Anorexia nervosa (binge/pu & & & \multicolumn{2}{|l|}{9} & 5.2 \\
\hline Other Specified Feeding or & er $(\mathrm{OS}$ & & \multicolumn{2}{|l|}{24} & 13.9 \\
\hline Self-harm reported at asses & & & \multicolumn{2}{|l|}{35} & 20.2 \\
\hline
\end{tabular}

routinely in the clinics (see Table 1 for details). To minimise bias arising from complete case analysis, we used multiple imputation to impute missing values.

\section{Multiple imputation}

We analysed patterns of missingness for baseline covariates, Time 2 alliance scores, and percentage median body mass index at 9 months by creating new binary variables to indicate completeness/missingness for each variable of interest. Binary logistic regression was used to identify significant predictors of missingness. We used multiple imputation with chained equations [39] and entered the following significant predictors of missingness: the EDE-Q Global score; the RCADS Internalising total score; adolescent Attachment Style Questionnaire Confidence and Preoccupation scales; the adolescent Difficulties in Emotion Regulation Scale Total score; the Reflective Function Scale-Youth total score, and the parental Attachment Style Questionnaire Preoccupation Scale. Auxiliary variables were age, baseline percentage median body mass index, site, sex, outcome, and use of higher level of care.

We imputed missing baseline covariate, Time 2 alliance scores, and 9-month \%mBMI median body mass index, using the MI impute chained command in Stata and 50 imputed datasets. All reported analyses of the primary and secondary outcome have been run using multiple imputation. All analyses were conducted in Stata v.15 (StataCorp, College Station, TX).

\section{Results}

\section{Demographics}

We recruited 192 adolescents and their parent/s to the study. Of this total, we excluded 19 participants (9.9\% of the sample) for the following reasons: 12 participants did not complete any measures for the study; two participants withdrew their consent; four were recruited to the study in error without meeting inclusion criteria; one participant had baseline data but no outcome data. The recruitment flowchart for the study is presented in Fig. 2. Recruitment took place from September 2015 to July 2018 in Site 1; February 2016 to July 2018 in Site 2; and January 2017 to July 2018 in Site 3.

The final sample for whom baseline and outcome data were available comprised 173 adolescents, of whom 153 $(88.4 \%)$ were female, with a mean age of 14.7 years. The parent sample comprised $n=163$, of whom 14 parents 


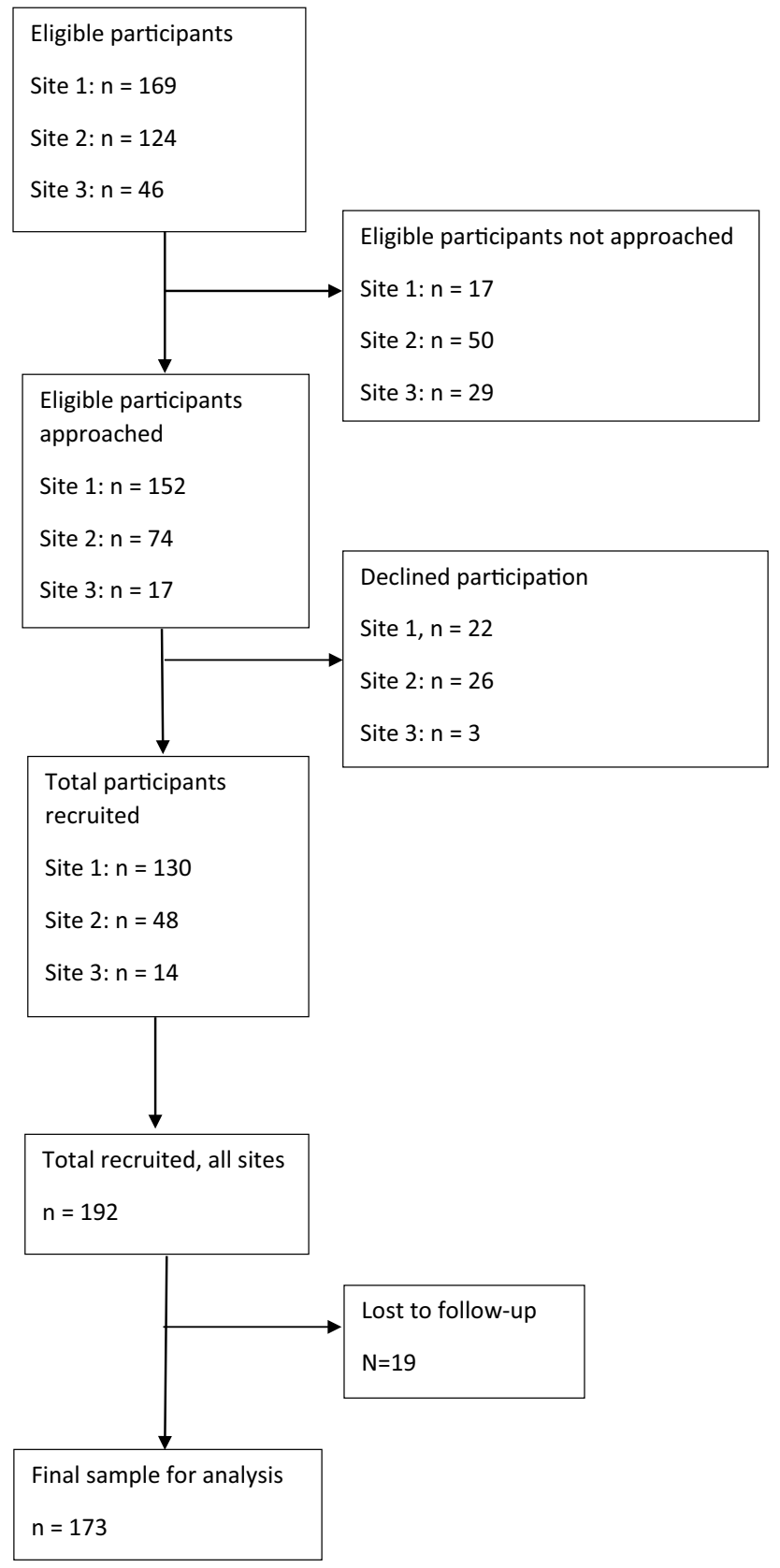

Fig. 2 Recruitment flowchart

were fathers and 149 parents were mothers. No parent data were available for ten adolescent participants. For 17 participants, data were available from both parents, as both had been recruited to the study. Since the majority of the parent sample were mothers, and to prevent artificially inflating the sample size beyond the 173 adolescents in the study, we excluded data from fathers in the 17 cases where mother data were available. Data from fathers were used for 14 adolescents who did not have data from mothers. 118 participants came from Site 1, 43 from Site
2, and 12 from Site 3. Demographic data for the sample are presented in Table 1. Thirty-four adolescents (19.7\%) attended some form of higher level of care, including 18 $(10.4 \%)$ who attended day-patient care, 11 (6.4\%) who were admitted to inpatient psychiatric care, and $11(6.2 \%)$ who were admitted to a paediatric ward.

\section{Correlations between predictor variables}

Table S1 reports Pearson's correlations between predictor variables.

\section{Descriptive statistics}

The range, means, and standard deviations for self-report measures are presented in Table 2 .

\section{Prediction of outcome from baseline covariates}

A higher baseline \%mBMI (OR $=1.11, \mathrm{CI}: 1.04-1.18)$ and higher self-reported eating pathology (EDE-Q Global score) $(\mathrm{OR}=1.36, \mathrm{CI}: 1.01-1.83)$ were associated with higher odds of positive outcome. Older age (OR: 0.63, CI: 0.48-0.82) and longer duration of illness (OR: 0.97, CI: 0.94-1.00) were associated with higher odds of poor outcome.

\section{Associations between predictors and outcome}

The associations between all predictor variables with treatment outcome, as well as associations between all $\mathrm{T} 1$ and $\mathrm{T} 2$ predictors, are available in Tables $\mathrm{S} 2$ and $\mathrm{S} 3$ for parents and adolescents, respectively. In the following sections, we highlight the significant findings for each pathway.

\section{Prediction of outcome from baseline variables}

Of the parent measures, higher scores on the RFQ8 Certainty subscale were predictive of poor outcome $(\mathrm{OR}=0.42$, CI: 0.20-0.87), whilst higher scores on the DERS Impulsive subscale $(\mathrm{OR}=1.23$, CI: 1.07-1.43) and Hypermentalizing Questionnaire (OR=1.03, CI: 1.00-1.06) predicted positive outcome. Of the adolescent measures, higher scores on the DERS Lack of Clarity scale, in which higher scores represent being unclear about one's feelings, predicted positive outcome $(\mathrm{OR}=1.10$, CI: $1.00-1.21)$.

\section{Prediction of outcome by alliance}

Of the SOFTA subscales, positive outcome was predicted by parental Emotional Connection score $(\mathrm{OR}=1.32$, CI: $1.09-1.58)$, parental Sense of Safety score $(\mathrm{OR}=1.24$, CI: 1.02-1.50), and the adolescent Shared Sense of Purpose score $(\mathrm{OR}=1.18, \mathrm{CI}: 1.01-1.38)$. 
Table 2 Range, means, standard deviations, and internal reliability of self-report measures

\begin{tabular}{|c|c|c|c|c|c|c|}
\hline Adolescents & $N$ & Min & Max & Mean & SD & Omega \\
\hline RCADS Total Internalising (T1) & 127 & 3 & 136 & 55.21 & 27.33 & 0.97 \\
\hline EDE-Q Global (T1) & 159 & .00 & 5.70 & 3.35 & 1.56 & 0.95 \\
\hline ASQ Confidence (T1) & 170 & 9 & 48 & 28.88 & 7.25 & 0.86 \\
\hline ASQ Discomfort (T1) & 170 & 10 & 58 & 39.83 & 8.86 & 0.88 \\
\hline ASQ Secondary (T1) & 170 & 7 & 38 & 19.59 & 5.94 & 0.80 \\
\hline ASQ need for approval (T1) & 170 & 11 & 42 & 30.44 & 5.81 & 0.77 \\
\hline ASQ Preoccupation (T1) & 170 & 8 & 46 & 29.82 & 6.70 & 0.76 \\
\hline DERS Nonacceptance (T1) & 170 & 6 & 30 & 18.60 & 7.56 & 0.94 \\
\hline DERS Goals (T1) & 170 & 5 & 25 & 17.98 & 5.20 & 0.89 \\
\hline DERS Impulse (T1) & 170 & 6 & 30 & 17.62 & 6.82 & 0.92 \\
\hline DERS Awareness (T1) & 170 & 6 & 30 & 17.62 & 5.10 & 0.81 \\
\hline DERS Strategies (T1) & 170 & 8 & 40 & 25.78 & 8.62 & 0.93 \\
\hline DERS Clarity (T1) & 170 & 5 & 25 & 14.58 & 4.98 & 0.89 \\
\hline HMZ (T1) & 169 & 2 & 94 & 53.56 & 18.41 & 0.92 \\
\hline RFQY (T1) & 170 & 6.26 & 10.44 & 8.78 & .79 & 0.76 \\
\hline SOFTA engagement (T2) & 125 & 4 & 20 & 12.36 & 3.62 & 0.75 \\
\hline SOFTA connection (T2) & 125 & 4 & 20 & 14.34 & 4.00 & 0.84 \\
\hline SOFTA safety (T2) & 125 & 4 & 20 & 11.52 & 3.70 & 0.70 \\
\hline SOFTA purpose (T2) & 125 & 6 & 20 & 15.04 & 3.32 & 0.72 \\
\hline Parents & $\mathrm{N}$ & Min & Max & Mean & $\mathrm{SD}$ & Omega \\
\hline ASQ Confidence (T1) & 163 & 12 & 47 & 36.06 & 5.58 & 0.82 \\
\hline ASQ Discomfort (T1) & 163 & 11 & 53 & 31.93 & 8.41 & 0.88 \\
\hline ASQ Secondary (T1) & 163 & 7 & 28 & 14.57 & 4.62 & 0.72 \\
\hline ASQ need (T1) & 162 & 7 & 36 & 20.73 & 5.60 & 0.78 \\
\hline ASQ Preoccupation (T1) & 163 & 11 & 47 & 24.67 & 6.67 & 0.81 \\
\hline DERS Nonacceptance (T1) & 163 & 6 & 30 & 11.42 & 5.14 & 0.88 \\
\hline DERS Goals (T1) & 163 & 5 & 25 & 12.56 & 4.50 & 0.86 \\
\hline DERS Impulse (T1) & 163 & 6 & 28 & 10.15 & 4.38 & 0.89 \\
\hline DERS Awareness (T1) & 163 & 6 & 26 & 14.49 & 4.37 & 0.79 \\
\hline DERS Strategies (T1) & 163 & 8 & 39 & 13.83 & 5.43 & 0.88 \\
\hline DERS Clarity (T1) & 163 & 5 & 20 & 8.79 & 2.92 & 0.80 \\
\hline RFQ8 Certainty (T1) & 163 & .00 & 3.00 & 1.22 & .74 & 0.79 \\
\hline RFQ8 Uncertainty (T1) & 163 & .00 & 2.17 & .41 & .45 & 0.76 \\
\hline $\mathrm{HMZ}(\mathrm{T} 1)$ & 162 & 8 & 81 & 39.78 & 17.55 & 0.93 \\
\hline SOFTA Engagement (T2) & 132 & 8 & 20 & 15.86 & 2.65 & 0.67 \\
\hline SOFTA Connection (T2) & 132 & 7 & 20 & 15.77 & 2.83 & 0.77 \\
\hline SOFTA Safety (T2) & 132 & 8 & 20 & 15.97 & 2.76 & 0.66 \\
\hline SOFTA Purpose (T2) & 132 & 8 & 20 & 16.83 & 2.85 & 0.75 \\
\hline
\end{tabular}

$R C A D S$ Revised Child Anxiety and Depression Scale (RCADS); EDE- $Q$ Eating Disorder Examination Questionnaire; $A S Q$ Attachment Style Questionnaire; ASQ Discomfort ASQ Discomfort with Closeness; ASQ Preoccupation ASQ Preoccupation with Relationships; ASQ Secondary ASQ Relationships as Secondary; DERS Difficulties in Emotion Regulation Strategies Scale; DERS Nonacceptance DERS Nonacceptance of Emotional Responses; DERS Goals DERS Difficulties Engaging in Goal-Directed Behaviours; DERS Impulse DERS Impulse Control Difficulties; DERS Awareness DERS Lack of Emotional Awareness; DERS Strategies DERS Limited Access to Effective Emotion Regulation Strategies; DERS Clarity DERS Lack of Emotional Clarity; RFQY Reflective Function Questionnaire - Youth Total Score; HMZ Hypermentalizing Questionnaire; SOFTA System for Observing Family Therapy Alliance; SOFTA Engagement SOFTA Engagement in the Therapeutic Process; SOFTA Connection SOFTA Emotional Connection to the Therapist; SOFTA Safety SOFTA Safety within the Therapeutic System; SOFTA Purpose SOFTA Shared Sense of Purpose within the Family; RFQ8 Reflective Function Questionnaire (8-item version); RFQ8 Certainty RFQ8 Certainty About Mental States; RFQ8 Uncertainty RFQ8 Uncertainty About Mental States. Omega refers to McDonald's Omega 


\section{Prediction of time 2 alliance by time 1 predictor variables}

Of the SOFTA subscales that were predictive of outcome, parental SOFTA Sense of Safety scores were predicted by parental ASQ Confidence ( $\beta=0.17$, CI: 0.07-0.26), parental ASQ Preoccupation ( $\beta=-0.11, \mathrm{CI}:-0.19$ to -0.03$)$, parental ASQ Need for Approval $(\beta=-0.10, \mathrm{CI}:-0.19$ to -0.01$)$, and parental DERS Goals subscale $(\beta=-0.15$, CI: -0.26 to -0.03$)$. No parental Time 1 predictor variables predicted parental SOFTA Emotional Connection scores.

For adolescents, the SOFTA Shared Sense of Purpose score was predicted by the adolescent DERS Awareness subscale $(\beta=-0.15, \mathrm{CI}:-0.28$ to -0.02$)$ and RFQY score $(\beta=1.02$, CI: 0.19-1.85).

\section{Secondary analysis: prediction of percentage median body mass index at 9 months}

No parent or adolescent predictor variables predicted $\%$ mBMI at 9 months.

\section{Discussion}

Our study investigated the hypothesis that attachment and mentalizing would predict alliance and outcome in FT-AN. Whilst the alliance was predictive of outcome in our data, and attachment and mentalizing were predictive of alliance, our theoretical model was only partially supported. Our results suggest that excessive certainty about mental states, a variable uncorrelated with alliance, was the strongest predictor of poor outcome, with each unit increase on the RFQ8 Certainty scale reducing the odds of a good outcome by more than half. For adolescents, higher scores on the DERS Lack of Clarity subscale also emerged as a predictor of outcome. Whilst this finding was in the opposite direction to our hypotheses, it is consistent with our findings for parents, since extreme clarity about one's feelings is conceptually similar to certainty about mental states, and indeed, we found that parental RFQ8 Certainty and adolescent DERS Lack of Emotional Clarity scores are negatively correlated in our data $(r=-0.28, p<0.01)$. In terms of possible mediating mechanisms, we speculate that excessive certainty might correlate with rigidity and difficulties in considering alternative perspectives. This could hamper recovery through reliance on a limited repertoire of problem-solving approaches.

In our PPI consultation, it was suggested that a high sense of clarity about one's feelings might be associated with a sense of certainty deriving from the eating disorder, and perhaps an identification with the 'eating disorder voice' which is associated with markers of severity such as longer duration of illness [40]. This perspective is congruent with the notion that ineffective mentalizing might influence outcome through lower levels of epistemic trust [41], resulting in reduced openness to learning in therapy. Relatedly, within the family context, excessive certainty on the part of the adolescent might also hamper their openness to accepting support from parents and might lead to a circular process whereby parental attempts to promote change are met with increased psychological rigidity on the part of the adolescent.

The association between outcome and the parental SOFTA Emotional Connection and Safety scales is in keeping with themes from qualitative studies which emphasise the importance for parents of trusting and feeling understood by their therapist $[42,43]$. Wallis et al. [42] have described a therapeutic process in FT-AN that they term relational containment, whereby treatment factors including the structured nature of the treatment, specialist treatment expertise, and consistent support create a sense of stability for parents and an expectation of progress, which helps to give them the confidence to validate their child's experiences and, at the same time, provide a supportive, predictable environment conducive to change. This then helps to establish a virtuous circle whereby increased parental confidence helps their adolescent to feel more secure, thereby promoting positive changes in eating behaviours as well as improved communication. This is consistent with our finding that the adolescent ratings on the SOFTA Shared Sense of Purpose were also predictive of a positive outcome. Our findings support the notion that the development of this sense of relational containment in the family may represent an important early treatment goal, which, from the perspective of adolescents, might contribute to establishing a shared sense of purpose for therapy. Notably, secure attachment and higher reflective function in adolescents were both predictive of positive alliance ratings, suggesting that these variables merit inclusion in future studies of alliance formation in FT-AN, such as studies incorporating measures of social and work functioning, session-by-session data collection, and observational measures of alliance.

Our study has several limitations, such as the use of self-report methods for both Time 1 and Time 2 predictors, thereby raising the issue of single-method variance. Furthermore, our measures of mentalization are relatively new, and have not yet been validated in adolescent eating disorder samples. Feedback from PPI activities highlighted that our choice of outcome was too reliant on weight as a criterion for success and did not include broader themes, such as positive indicators of well-being, which have been shown to be important to recovery from the perspective of those with lived experience [44]. 


\section{Conclusion}

In summary, our data are the first to suggest that mentalizing difficulties may predict poor outcome in FT-AN, particularly when present in parents. Study strengths include the relatively large sample drawn from three different sites providing specialist out-patient treatment, thereby increasing the external validity of the study. Other strengths include the use of PPI to inform both method and interpretation. Future research should use multi-method approaches to investigate processes of change, including the roles of mentalizing and alliance, in studies using broader definitions of recovery that are salient to parents and adolescents with lived experience of eating disorders.

Supplementary Information The online version contains supplementary material available at https://doi.org/10.1007/s00787-021-01930-3.

Author contributions TJ, PF, and IE designed the study and wrote the fellowship application which was funded by the National Institute for Health Research (NIHR). MH contributed to the analysis plan. TJ conducted the statistical analysis in consultation with $\mathrm{MH}, \mathrm{PF}$, and IE. LS, AE, and MS were involved in the acquisition of study data. TJ drafted the manuscript with critical revision from MH, LS, AE, MS, PF, and IE. All authors have approved the final article.

Funding This study was supported by National Institute of Health Research (NIHR) Clinical Doctoral Research Fellowship, Tom Jewell, CDRF-2014-05-024. Dr Moritz Herle is funded by a fellowship from the Medical Research Council UK (MR/T027843/1).

Availability of data and material Data are available from the corresponding author on request.

Code availability Code is available from the corresponding author on request.

\section{Declarations}

Conflict of interest The authors declare that they have no conflict of interest.

Ethical approval All procedures performed were in accordance with the ethical standards of the institutional research committee and with the 1964 Helsinki declaration and its later amendments or comparable ethical standards.

Consent to participate Not applicable/covered by ethical approval.

Consent for publication Not applicable.

Open Access This article is licensed under a Creative Commons Attribution 4.0 International License, which permits use, sharing, adaptation, distribution and reproduction in any medium or format, as long as you give appropriate credit to the original author(s) and the source, provide a link to the Creative Commons licence, and indicate if changes were made. The images or other third party material in this article are included in the article's Creative Commons licence, unless indicated otherwise in a credit line to the material. If material is not included in the article's Creative Commons licence and your intended use is not permitted by statutory regulation or exceeds the permitted use, you will need to obtain permission directly from the copyright holder. To view a copy of this licence, visit http://creativecommons.org/licenses/by/4.0/.

\section{References}

1. Salbach-Andrae $\mathrm{H}$ et al (2008) Psychiatric comorbidities among female adolescents with anorexia nervosa. Child Psychiatry Hum Dev 39:261-272

2. Arcelus J, Mitchell AJ, Wales J, Nielsen S (2011) Mortality rates in patients with anorexia nervosa and other eating disorders: a meta-analysis of 36 studies. Arch Gen Psychiatry 68:724-731

3. Petkova $\mathrm{H}$ et al (2019) Incidence of anorexia nervosa in young people in the UK and Ireland: a national surveillance study. BMJ Open 9:e027339

4. National Institute for Health and Care Excellence (2017) Eating disorders: recognition and treatment. NG69

5. Hamadi L, Holliday J (2020) Moderators and mediators of outcome in treatments for anorexia nervosa and bulimia nervosa in adolescents: a systematic review of randomized controlled trials. Int J Eat Disord 53:3-19

6. Jewell $\mathrm{T}$ et al (2016) Attachment and mentalization and their association with child and adolescent eating pathology: a systematic review. Int J Eat Disord 49:354-373

7. Doyle PM, Grange DL, Loeb K, Doyle AC, Crosby RD (2010) Early response to family-based treatment for adolescent anorexia nervosa. Int J Eat Disord 43:659-662

8. Isserlin L, Couturier J (2012) Therapeutic alliance and familybased treatment for adolescents with anorexia nervosa. Psychotherapy 49:46-51

9. Simic M, Jewell T, Eisler I (2020) Beneath the surface of expressed emotion (EE): the clinical relevance of possible mechanisms underlying EE in eating disorders. Eating disorders and expressed emotion. Taylor and Francis, UK, pp 33-57

10. Bowlby J (1969) Attachment and loss. Basic Books, New York

11. Ainsworth MDS, Blehar MC, Waters E, Wall SN (1978) Patterns of attachment: a psychological study of the strange situation. Erlbaum, New Jersey

12. Fraley RC, Roisman GI (2019) The development of adult attachment styles: four lessons. Curr Opin Psychol 25:26-30

13. Caglar-Nazali HP et al (2014) A systematic review and metaanalysis of 'Systems for Social Processes' in eating disorders. Neurosci Biobehav Rev 42:55-92

14. Bernecker SL, Levy KN, Ellison WD (2014) A meta-analysis of the relation between patient adult attachment style and the working alliance. Psychother Res 24:12-24

15. Levy KN, Kivity Y, Johnson BN, Gooch CV (2018) Adult attachment as a predictor and moderator of psychotherapy outcome: a meta-analysis. J Clin Psychol 74:1996-2013

16. Folke S, Daniel SIF, Poulsen S, Lunn S (2016) Client attachment security predicts alliance in a randomized controlled trial of two psychotherapies for bulimia nervosa. Psychother Res 26:459-471

17. Tasca GA, Balfour L (2014) Attachment and eating disorders: a review of current research. Int J Eat Disord 47:710-717

18. Fonagy P, Steele M, Steele H, Moran GS, Higgitt AC (1991) The capacity for understanding mental states: the reflective self in parent and child and its significance for security of attachment. Infant Ment Health J 12:201-218

19. Luyten P, Campbell C, Allison E, Fonagy P (2020) The mentalizing approach to psychopathology: state of the art and future directions. Annu Rev Clin Psychol 16:297-325 
20. Katznelson $\mathrm{H}$ et al (2020) Reflective functioning, psychotherapeutic alliance, and outcome in two psychotherapies for bulimia nervosa. Psychotherapy 57:129-140

21. Rothschild-Yakar L, Levy-Shiff R, Fridman-Balaban R, Gur E, Stein D (2010) Mentalization and relationships with parents as predictors of eating disordered behavior. J Nerv Ment Dis 198:501-507

22. Long M, Verbeke W, Ein-Dor T, Vrtička P (2020) A functional neuro-anatomical model of human attachment (NAMA): insights from first- and second-person social neuroscience. Cortex. https://doi.org/10.1016/j.cortex.2020.01.010

23. Fonagy P, Luyten P (2009) A developmental, mentalizationbased approach to the understanding and treatment of borderline personality disorder. Dev Psychopathol 21:1355-1381

24. White HJ, Haycraft E, Williamson I, Meyer C (2020) Disturbance at the dinner table: exploring mothers' experiences of mealtimes when caring for their son or daughter with anorexia nervosa. J Health Psychol. 1359105320904756. https://doi.org/ $10.1177 / 1359105320904756$

25. Sharp C et al (2011) Theory of mind and emotion regulation difficulties in adolescents with borderline traits. J Am Acad Child Adolesc Psychiatry 50:563-573.e1

26. Graves TA et al (2017) A meta-analysis of the relation between therapeutic alliance and treatment outcome in eating disorders. Int J Eat Disord 50:323-340

27. Flückiger C, Del Re AC, Wampold BE, Horvath AO (2018) The alliance in adult psychotherapy: a meta-analytic synthesis. Psychotherapy 55:316

28. American Psychiatric Association (2013) The diagnostic and statistical manual of mental disorders: DSM-5, 5th edn. American Psychiatric Association, USA

29. Child Growth Foundation (1990) UK cross-sectional reference data: 1990/1. Child Growth Foundation, London

30. Russell GF, Szmukler GI, Dare C, Eisler I (1987) An evaluation of family therapy in anorexia nervosa and bulimia nervosa. Arch Gen Psychiatry 44:1047-1056

31. Feeney JA, Noller P, Hanrahan M (1994) Assessing adult attachment. Attachment in adults: clinical and developmental perspectives. Guilford Press, New York, pp 128-152
32. Gratz KL, Roemer L (2004) Multidimensional assessment of emotion regulation and dysregulation: development, factor structure, and initial validation of the difficulties in emotion regulation scale. J Psychopathol Behav Assess 26:41-54

33. Dziobek I et al (2006) Introducing MASC: a movie for the assessment of social cognition. J Autism Dev Disord 36:623-636

34. Ha C, Sharp C, Ensink K, Fonagy P, Cirino P (2013) The measurement of reflective function in adolescents with and without borderline traits. J Adolesc 36:1215-1223

35. Fonagy $\mathrm{P}$ et al (2016) Development and validation of a self-report measure of mentalizing: the reflective functioning questionnaire. PLoS ONE 11:e0158678

36. Friedlander ML et al (2006) System for observing family therapy alliances: a tool for research and practice. J Couns Psychol 53:214-225

37. Fairburn CG, Beglin SJ (1994) Assessment of eating disorders: interview or self-report questionnaire? Int J Eat Disord 16:363-370

38. Chorpita BF, Yim L, Moffitt C, Umemoto LA, Francis SE (2000) Assessment of symptoms of DSM-IV anxiety and depression in children: a revised child anxiety and depression scale. Behav Res Ther 38:835-855

39. Royston P, White IR (2011) Multiple imputation by chained equations (MICE): implementation in stata. J Stat Softw 45:1-20

40. Pugh M, Waller G (2017) Understanding the 'anorexic voice' in anorexia nervosa. Clin Psychol Psychother 24:670-676

41. Fonagy P, Luyten P, Allison E (2015) Epistemic petrification and the restoration of epistemic trust: a new conceptualization of borderline personality disorder and its psychosocial treatment. J Personal Disord 29:575-609

42. Wallis A et al (2017) Relational containment: exploring the effect of family-based treatment for anorexia on familial relationships. J Eat Disord 5:27

43. Williams LT, Wood C, Plath D (2020) Parents' experiences of family therapy for adolescent anorexia nervosa. Aust Soc Work 73:408-419

44. Bohrer BK, Foye U, Jewell T (2020) Recovery as a process: exploring definitions of recovery in the context of eating-disorderrelated social media forums. Int J Eat Disord 53:1219-1223 